\title{
LES NOMS DES SAINTS \& IMAGINAIRES ET FACÉTIEUX $\gg$ EN HAGIOTOPONYMIE FRANÇAISE
}

\author{
Michel A. Rateau \\ Université du Temps Libre (UTL), Bergerac, France
}

\begin{abstract}
Names of "imaginary and facetious" saints in French hagiotoponymy
Abstract: Contrary to what might be thought, few believers or non-believers are aware that, among the place names including the onym of a saint or hagiotoponyms, many do not represent the least holy man or the least holy woman. As will be shown, some of these names have been substituted for names of actual saints (who actually lived), formerly popularly created by our Christian ancestors in a facetious spirit or sometimes in all seriousness. Others, skilful users of slang or the "green tongue", members of the brotherhood of gaiety and Bacchus, invented and then popularized other imaginary saints. Sometimes surprisingly, these saints coexist along "true saints" and the "humbler people" who believe in them. Their poetic, colourful nature reveals the pleasant popular flavour of the centuries of another age.
\end{abstract}

Keywords: false saints, imaginary, facetious, hagiotoponymy, hagiomicrotoponymy.

\section{0 - Introduction}

Après avoir donné un bref mais nécessaire éclairage sur ce qu'il convient d'appeler des noms de « saints imaginaires et facétieux en hagiotoponymie française $»$, seront expliquées les principales étapes de méthodologie mises en œuvre afin de les identifier et de les localiser. Enfin, sera développé ce sujet français, de par le territoire national concerné, au moyen d'exemples produits en langues bretonne, d'oc (occitan) ou d'oül.

\section{1 - De quelques définitions et autres explications}

\section{1 - « Saints imaginaires 》}

Pour l'Église catholique, apostolique et romaine, pour être un saint ou une sainte officiellement reconnu /-e, il faut que ce saint personnage ait réellement existé, ce qui n'est pas le cas de ceux mis en scène dans ce sujet.

« Imaginaires » signifie qu'ils ont purement et simplement été créés par l'imagination des hommes et des femmes, de toute pièces, soit par besoin, soit incidemment, mais qu'ils ne peuvent en aucun cas exister comme « saints $\gg /$ saintes $\gg$, au sein de ladite Église, même lorsque cela semble être le cas contraire au sein de telle ou telle autre communauté populaire et de ses croyances.

En théorie au moins, les dévotions qui leur sont accordées, ne sont pas reconnues 
pas ladite Église bien que, dans le monde profane, certains de ses « cadres » (curés, desservants, prêtres...) aient pu fermer les yeux sur certaines pratiques, 'ni catholiques ni orthodoxes', parfois même superposées comme ayant appartenu à de très anciens rites païens.

On ne confondra ni avec les saints « hypothétiques » (Darchen 2017: 309), auxquels il est plutôt aventureux d'attribuer une 'identité chrétienne' (saint Pompon, saint Marcory...) ni les saints qui n'ont pour origine qu'une erreur d'orthographe ou une liaison aussi involontaire que malencontreuse ( ${ }^{*}$ saint Drieux pour Cendrieux (24), saint Honger ou saint Onger (24) pour Saintonger qui signifie 'originaire de l'ancienne province de Saintonge)...

1.2 - « Facétieux » reflète, ici, un esprit de truculence, de plaisanterie, de moquerie, de malice, sans, toutefois, être violemment empreint d'anticléricalisme.

1.3 - Pour être étudiés dans cet article, il ne leur suffit pas de compter parmi ces « pseudo-saint(e)s , il faut également que ces «pseudo-hagionymes » participent à la composition de noms de lieux, actuels ou passés voire totalement disparus, et ce dans d'apparents hagiotoponymes / microhagiotoponymes / nanohagiotoponymes. Les noms de saints imaginaires et facétieux sont innombrables (Merceron 2002), mais fort peu appartiennent au domaine toponymique: ici aussi, 'beaucoup d'appelés, bien peu d'élus'...

1.4 - Il vient d'être parlé de hagiotoponymes / microhagiotoponymes / nanohagiotoponymes: on le verra, certains d'entre eux peuvent, simultanément, appartenir à d'autres sous-catégories. Ainsi, c'est le cas des noms de sources et de ceux des fontaines sacrées ou miraculeuses, etc., qui sont également des hagiohydronymes, /microhagiohydronymes / nanohagiohydronymes. Cependant, développer ici ce phénomène onomastique nous entraînerait hors de notre sujet.

1.5 - Leurs auteurs ou créateurs, inconnus pour l'immense majorité d'entre eux, sont issus du monde populaire. Cependant, au cours des âges, certains écrivains, plus connus de leur temps, ont prêté leur plume à ce genre parfois littéraire...

\section{2 - Méthodologie bibliographique et services d'archives}

Tout d'abord, comment ne pas avoir utiliser, pour base principale, le Dictionnaire des saints imaginaires et facétieux, de Jacques E. Merceron, paru aux éditions du Seuil, à Paris, en 2002? Il a d'abord fallu puiser dans ses 1275 pages, bien commentées et référencées, et établir la liste des toponymes recherchés.

Puis, à l'aide de l'Internet ou par téléphone auprès des secrétariats municipaux (registres de délibérations) et / ou services d'archives (registres et plans cadastraux) des mairies concernées, il a été utile et nécessaire d'essayer de vérifier les données plus ou moins légendaires qui ont été publiées. 
Ensuite, des vérifications de localisation ont pu être effectuées grâce au logiciel du Dictionnaire des Toponymes de France du CDIP (http//wwwcdip.com), dont les données cartographiques intégrées sont la propriété de l'Institut National de l'information Géographique et forestière (IGN). On peut également tenter de vérifier ces diverses données avec les cartes de Belleyme ou de Cassini mais ces dernières sont trop rarement suffisamment détaillées pour être utiles.

Enfin, de nouveau à l'aide de l'Internet, il était nécessairement complémentaire de rechercher des textes ou témoignages d'auteurs, pouvant confirmer ou infirmer, transversalement, les petites histoires, légendes et autres anecdotes racontées et recueillies.

\section{3 - Les hagiotoponymes / microhagiotoponymes / nanohagiotoponymes}

On le verra, par opposition aux choronymes ainsi qu'aux toponymes correspondants à des lieux d'importance moyenne (communes voire lieux-dits, bâtis ou non), ces noms de lieux inventés semblent, la plupart du temps, pouvoir être regroupés au sein d'une sous-catégorie toponymique, généraliste en la matière, celle des «noms de très petits lieux-dits », réunissant à la fois, ici, les faux microhagiotoponymes (noms de chapelles, de fontaines bâties) et les faux nanohagiotoponymes (noms de lieux d'érection de statues de pèlerinage, de dévotions diverses, de sources non bâties), tous populairement inventés, connus et parfois diversement nommés.

Afin de donner un ordre de grandeur, notons qu'en Bretagne seule, plus de 2.000 fontaines sacrées ont été recensées (Oratoires: site net), mais aussi que bien peu d'entre elles sont dédiées à / et portent un nom de saint(e) imaginaire, protecteur ou guérisseur.

Dans notre classement de présentation (numéroté mais sans notion d'exhaustivité), nous avons décidé de privilégier une sélection d'exemples mettant en avant l'idée de croyance(s) imaginaire(s) et / ou miraculeuse(s) liés à la santé des humains, plutôt que celles de toponymie ou d'hydronymie.

\section{1 - Les 'médecins généralistes': saints protecteurs / guérisseurs d'adultes}

À Cissé (Vienne), naguère, le 22 septembre, on vénérait une statue dédiée à Saint Violet, qui, en fait, était saint Sylvain, dont la fête est bien ce jour-là. Il y a ici un cas de substitution: le remplacement d'un saint reconnu par l'Église, par un saint imaginaire, fabriqué de toute pièces, selon diverses circonstances. En effet, des pèlerins qui désiraient ardemment éviter d'être atteints du mal violet (coloration de la peau pour cause de cyanose) ou bien pour s'en faire soigner, lors de / ou après un ou plusieurs pèlerinages, accrochaient à ladite statue des rubans violets contre ledit mal (Merceron 2002: 524). On ne doit pas, ici, considérer cette statuette comme un objet mais comme un nom de lieu cultuel: on ne se rendait pas dans une église mais au lieu de la Statue de Saint-Violet.

À La Puye (Vienne), se trouve une Fontaine de Saint-Bonifet, un présumé saint homme qui n’a jamais existé, et on attendait de cet être que, en effet, il fasse (du) 
bien (« bon / bien-y / il-fait »)! Il s'agit de l'une des représentations collectives du «Bienfaisant 》 (Merceron 2002: 68). Il a pu se substituer à une ancienne divinité ondine, de quelque sorte d'eau, plus ou moins courante, d'une résurgence, d'une fontaine aménagée ou non, ou bien rappeler une dévotion préchrétienne, à « quelque créateur ».

\section{2 - 'Gynécologues et sages-femmes': des assistantes pour de meilleures grossesses}

À Bertric-Burée (Dordogne), se trouve la Fontaine Sainte-Mamelée, aux propriétés curatives mal précisées ou mal comprises selon les interprétations populaires et / ou scientifiques (Merceron 2002: 267; Rigouste 2009: 3). Les mamans en difficulté de lactation et ne pouvant allaiter leur dernier rejeton, s'y rendaient avec toute la ferveur et l'espoir que l'on imagine. Il arrivait également que des nourrices essayent, à leur tour, de profiter de la chance qui pouvait leur être offerte alors qu'elles risquaient de mettre en danger leur activité professionnelle.

À Bouillé-Loretz (Deux-Sèvres), se découvre une Statue (de) dédiée à SaintBandard (Merceron 2002: 161) au nom populairement et argotiquement révélateur tout comme son étymologie ( < francique ${ }^{*}$ binda, lien, destiné à être tendu ...) (Dubois 1977: 152; Lambrechts 2001: 85), dont le culte qui lui était accordé provoquait chaque année un pèlerinage très suivi auquel participaient les femmes 'brehaignes' (stériles) qui désiraient avoir des enfants et nombre d'hommes « près desquels les sourires de Vénus n'avaient plus guère d'efficacité... » (id.). La base de l'adjectif brehaigne, dont l'origine demeure 'obscure', a produit la suite: baraigne (ancien français, 1119), barai (g) ne (vieux français), barhai $(g) n e$, brahai $(g) n e$ (moyen français, après rhotacisme), brehai (g)ne, bréhaigne (Onions 1983: 76; Dubois 1977: 152, 217). L’anglo-normand (XIe - XIIIe s.) est à l'origine de l'anglais barren, porteur du même sens (Onions 1983: 76).

Nous ne nous étendrons pas sur les innombrables noms de très petits lieux dénommant des menhirs et autres pierres dressées, souvent tels de fiers mais légendaires phallus après desquelles se rendaient, seules ou à l'occasion de pèlerinages, d'innombrables dames et autres demoiselles, ce qui se peut comprendre, et, peut-être par suite d'un phénomène social urologique moins connu, tout autant de damoiseaux et autres grands seigneurs aux problèmes divers et inavoués... Et pour faire ici court et simple, que de saint(e)s furent supplié(e)s: Aboutit, Badine, Bandouille, Bedoche (au 'bon effet', on lui embrassait le nombril, etc.), Bidault, Birottin (Birontin, Biroton, Biroutin...), Biroute (qui rendait les femmes si fécondes...), Bitochon, Sainte-Blessure (invoquant plus poétiquement les 'menstrues')... (Merceron 2002: 151 à 324). Et nous n'en sommes qu'à la lettre $\ll B \gg \ldots$

\section{3 - Les 'pédiatres': guérisseurs d'enfants}

Sur la commune de Trénez (Finistère), se trouve la Fontaine Sainte-Ventroc où cette « sainte » est invoquée afin de guérir les 'ventres' tourmentés (Merceron 2002: 
522). Son étymologie populaire régionale paraît très convaincante: en breton, gwentr signifie 'colique'... Le nom de cette sainte est sémantiquement fabriqué selon le besoin.

Autrefois, La Croix (de) Sainte-Baudruche, sise à l'un des carrefours des Rosierssur-Loire (Maine-et-Loire), passait pour soigner de la « coqueluche » (Merceron 2002: 523). Simultanément à cette forme de déclaration d'espoir caché, on observe un cas d'amusement phonétique populaire, rimant en -uche.

En Corrèze (Saint-Cirgues-la-Loutre), on se rendait, autrefois, à La Fon SentEstropi (forme occitane d'une *Fontaine Saint-Estropié, non attestée en langue d'oil), pour y implorer ledit saint (Merceron 2002: 594) afin qu'il permette aux très jeunes (et aux moins jeunes...) enfants « en retard de marcher », d'enfin mieux trotter voire galoper.

\section{4 - Les 'pneumologues' d'antan}

Un autre pèlerinage, cette fois à Courtalain (Eure-et-Loire), a donné vie à une imaginaire Sainte-Courte-Haleine ('courte' + alaine = haleine), laquelle devait, avec plein succès, si possible, participer à la disparition des crises d'un asthme que, d'ailleurs, sur ce même lieux, on y soigne encore de nos jours (Merceron 2002: 808). Les qualités curatives de ce lieu-dit semblent ne pas avoir été suffisantes et le peuple des malades fit appel à son imagination étymologique populaire et à son esprit créateur de jeux de mots approximatifs. Notons, cependant, que 'haleine' provient du latin halare, signifiant 'souffler', après s'être écrit aleine dans la Chanson de Roland, en 1080.

\section{5 - Les 'dermatologues': protecteurs contre / et guérisseurs de la teigne et la gale...}

En Belgique (francophone) et en France dans plusieurs régions, existait déjà saint Aignan (Merceron 2002: 518). À force de pratiquer plus que de raison une liaison (encore un amusement ou une dérive phonétique!) excessive, saint Aignan devint, verbalement, Saint-Teignan (et variantes) et ce nouveau saint, aussi imaginaire que facétieux, fut associé à la maladie de la teigne, aussi capillaire que dermique. «Teignan l'imaginaire » était alors invoqué pour la soigner. Phonétiquement, on vient de le voir, il y avait superposition, une sorte d'emprunt phonétique bien arrangeant.

Par contamination socio-onomastique, à la Celle-en-Morvan (Saône-et-Loire), la Fontaine Saint-Aignan guérissait de la gale, une autre maladie dermique (Merceron 2002: 517) Curieusement, ce saint perdit la dévotion populaire accordée à ce lieu-dit ondin, lorsqu'un paysan y conduisit son cheval galeux et que cet infortuné quadrupède ne rentra point guéri à la ferme... Croyances et déceptions populaires...

Dans l'Indre, à Saint-Aigny, existait une Fontaine Saint-Aigny (variante attestée d'Aignan) qui avait pour vertu celle de guérir les «taigneux $\gg \ldots$ ainsi que les croûtes de lait des nourrissons, l'eczéma et l'urticaire (Merceron 2002: 518). Il est ici évident qu'il s'agit des suites du même jeu d'esprit. 


\section{6 - Les saints guérisseurs des maladies ou maux de langueur}

Au hameau de Chenault, près Précy-sur-Thil (Côte-d'Or), jusqu'au XIXe s., était réputée une Fontaine miraculeuse dédiée à Saint-Élangueu qui lui en avait donné son nom, laquelle permettait de soigner ou de diminuer les maladies de langueur (Merceron 2002: 415). Il s'y déroulait alors un rite divinatoire. On étendait un linge sur l'eau après qu'il ait touché le corps d'un sujet atteint d'une maladie de langueur. Selon la légende locale, l'édicule qui y avait été construit à proximité, abritait une Statuette de SainteAlangueure. Il a pu s'agir de la continuation d'un ancien « culte des fontaines $\gg(i d$.). On relève ici une apparente polyvalence 'masculine - féminine' rappelant, peut-être, d'anciennes orientations païennes.

Autrefois, à Gouézev (Finistère), au hameau de Lanrigui, se trouvait une Chapelle avec Statue formant un trio miraculeux avec la Fontaine Sant-Ty-Poen, qui subsiste encore (Merceron 2002: 417). À Leulan (id.) était érigée la Chapelle du Gouellet aussi appelée Chapelle Sant-Ty-Poen; près de Quimperlé, c'était également une Chapelle de San-Ty-Poen ou encore Notre-Same-des-Peines-et-des-Souffrances, en fait, un nom de lieu qui traduit son nom breton. «Ty-Poen » est une variante du nom du « (saint) Diboan » imaginaire, qui dérive du verbe breton diaboania ('soulager les souffrances') (id.). Cette traduction est la variante française liturgiquement employée... (id.: 419).

\section{7 - Les 'ophtalmologues' des villes et des champs}

À Ruffiac (Lot-et-Garonne), autrefois, une source dédiée saint Rémi permettait à ce (véritable) saint de soigner (c'est ce qui était espéré...) les maux d'yeux. Les croyances populaires firent évoluer cet hagionyme en Source de Saint-Remède aussi connue, régionalement, sous la variante saint-Rémèdy ou sent-Remèdi, en occitan. La forme féminine, Sainte-Remède était également utilisée (Merceron 2002: 509; Rigouste et Gardou 2015: 47).

\section{4 - Quelques premières observations pré-conclusives}

Avec ces quelques exemples, on aura pu observer que cette sous-catégorie d'hagiotoponymes, parmi lesquels se comptent les microhagiohydronymes et les nanohagiotoponyme, objets de notre propos, est essentiellement constituée de noms de très petits lieux, où se trouvent une source (lieu non bâtie), une résurgence (parfois aménagée) ou une fontaine (lieu bâti), une petite chapelle, plus ou moins bien conservée, une statue ou statuette érigée dans une chapelle ou dans un modeste oratoire, l'un ou l'autre situé à l'extérieur, transportable / déplaçable ou fixe, taillée dans le roc ou la roche, ou encore des mégalithes comparés à de vénérables phallus bienfaiteurs. Socialement et religieusement, on note également que ces microtoponymes peuvent aussi être des lieux de fervents pèlerinages.

Un certain nombre ont disparu: ces noms de lieux, « hagionymisés », n' habitent plus que la mémoire des chercheurs et des auteurs (folkloristes et historiens). Un grand 
nombre rappelle des histoires socioreligieuses apparemment 'vraies', pourtant contées dans des légendes qui le sont moins.

Ils participent, aussi, au remplacement d'anciens noms de lieux païens, de dévotions préchrétiennes, de divinités oubliées, qui ne nous sont, pour la plupart, jamais parvenus. Dans d'autres cas, on parle de 'substitution' (Saint Violet remplace Saint Sylvain); de 'sacralisation d'objets' qui deviennent des 'lieux sacrés' (statues, rochers, mégalithes...).

Cet ensemble de 'prescripteurs bienfaisants' bien qu'appartenant au monde de l'incertain, entretenant l'espoir des petits comme de leurs parents, correspond plutôt bien au 'codex à la fois toponymique et hagionymique voire anthroponymique, mystérieux et religieusement ésotérique' que constituent ces «noms de lieux très petits ».

Les facéties et grivoiseries (Saint-Bandard), les rimes (Sainte-Baudruche avec coqueluche), les jeux phonétiques empruntés au lexique (Sainte-Courte-Haleine, SaintTy-Poen, Saint-Teignan), les traductions en langues régionales (breton, occitan, wallon...) ou encore les incertitudes régnant quant au sexe, non pas des anges mais des saint(e)s, pour ne rappeler que ces cas spécifiques, montrent combien le sujet est aussi plaisant à étudier et onomastiquement richissime.

\section{5 - En guise de conclusion}

Toponymiquement, tous ces 'noms de très petits lieux' ont un point commun, présent ou passé, celui de nommer un lieu, d'être un véritable toponyme, puisque l'on s'y rend! puisqu'il désigne une destination finale, un but à atteindre, aussi. Et en l'occurrence, un pseudo-hagiotoponyme.

Culturellement, ce qui retient aussi notre attention, c'est le fait même de la double fonction de 'pseudo-nom sacré' ('anthroponymie religieuse') et de 'nom de lieu' (toponymie naturelle, plus profane): désigner celui dont les vertus sont divinement liées tant à sa spécificité médicale qu'à la croyance populaire qui flotte dans son environnement, entre espoir de guérison due à un saint ou à une sainte, et nom du lieu (ici = adresse du médecin ou du spécialiste!) où la réalité des choses de la vie reste à découvrir.

Administrativement, il est curieux mais non moins intéressant d'observer de quelle façon des noms de lieux constitués à partir de « faux anthroponymes » peuvent devenir et deviennent « officiels », ou comment on peut passer du « non conventionnel $\gg$ au $\ll$ conventionnel $\gg$.

Certains des noms des saints imaginaires remplaceraient-ils les noms d'antiques et légendaires génies ondins « de toujours », d'anciennes divinités locales, gauloises (?), pré-aquitaines (?), pré-indoeuropéennes (?) si couramment évoquées en France, lorsque l'on n'en sait pas plus que cela? Nous laissons, à nos continuateurs, le soin de nous le faire découvrir. 


\section{Bibliographie}

Darchen, Bernadette. 2017. Les saints patrons du Périgord... des saints pas toujours très catholiques. Le Bugue: P.L.B. éditeur.

Dubois, Jean (sous la direction). 1977. Larousse de la langue française: lexis. Paris: Librairie Larousse.

Lambrechts, Chantal (direction d'édition). 2001. Dictionnaire historique et étymologique du français. Paris: Larousse.

Merceron, Jacques E. 2002. Dictionnaire des saints imaginaires et facétieux. Paris: Éditions du Seuil.

Onions, C. T. 1983. The Oxford Dictionary of English Etymology. Oxford: Clarendon Press.

Rigouste, Jean. 2009. Les saints imaginaires et facétieux, titre du polycopié du chapitre de son cours d'onomastique (4 pages). Université du Temps Libre (UTL) de Bergerac et de ses conférences (Bergerac / 24, Blaye / 33, Villeneuve-sur-Lot / 47). Texte non publié.

Rigouste, Jean et Charles Gardou. 2015. En Quercy, au handicap les saints remèdes. Ou celui qui croit sera guéri. Dans Le handicap dans notre imaginaire culturel, variations anthropologiques 2, Charles Gardou (ed.), 29-50. Toulouse: Èrès.

\section{Source électronique}

Oratoires: http://www.les-oratoires.asso.fr/fontaines-de-dévotion (vu 16.09.17). 